\title{
Purine biosynthesis-deficient Burkholderia mutants are incapable of symbiotic accommodation in the stinkbug
}

\author{
Jiyeun Kate Kim ${ }^{1}$, Ho Am Jang ${ }^{1}$, Yeo Jin Won ${ }^{1}$, Yoshitomo Kikuchi ${ }^{2}$, Sang Heum Han ${ }^{1}$, \\ Chan-Hee Kim ${ }^{1}$, Naruo Nikoh ${ }^{3}$, Takema Fukatsu ${ }^{4}$ and Bok Luel Lee ${ }^{1}$ \\ ${ }^{1}$ Global Research Laboratory, College of Pharmacy, Pusan National University, Pusan, South Korea; ${ }^{2}$ Bioproduction \\ Research Institute, National Institute of Advanced Industrial Science and Technology (AIST), Hokkaido Center, \\ Sapporo, Japan; ${ }^{3}$ The Open University of Japan, Department of Liberal arts, Chiba, Japan and ${ }^{4}$ Bioproduction \\ Research Institute, National Institute of Advanced Industrial Science and Technology (AIST), Tsukuba, Japan
}

\begin{abstract}
The Riptortus-Burkholderia symbiotic system represents a promising experimental model to study the molecular mechanisms involved in insect-bacterium symbiosis due to the availability of genetically manipulated Burkholderia symbiont. Using transposon mutagenesis screening, we found a symbiosis-deficient mutant that was able to colonize the host insect but failed to induce normal development of host's symbiotic organ. The disrupted gene was identified as purL involved in purine biosynthesis. In vitro growth impairment of the purL mutant and its growth dependency on adenine and adenosine confirmed the functional disruption of the purine synthesis gene. The purL mutant also showed defects in biofilm formation, and this defect was not rescued by supplementation of purine derivatives. When inoculated to host insects, the purL mutant was initially able to colonize the symbiotic organ but failed to attain a normal infection density. The low level of infection density of the purL mutant attenuated the development of the host's symbiotic organ at early instar stages and reduced the host's fitness throughout the nymphal stages. Another symbiont mutantdeficient in a purine biosynthesis gene, purM, showed phenotypes similar to those of the purL mutant both in vitro and in vivo, confirming that the purL phenotypes are due to disrupted purine biosynthesis. These results demonstrate that the purine biosynthesis genes of the Burkholderia symbiont are critical for the successful accommodation of symbiont within the host, thereby facilitating the development of the host's symbiotic organ and enhancing the host's fitness values. The ISME Journal (2014) 8, 552-563; doi:10.1038/ismej.2013.168; published online 3 October 2013
\end{abstract}

Subject Category: Microbe-microbe and microbe-host interactions

Keywords: purine biosynthesis; infection density; insect gut symbiosis; Burkholderia symbiont; Riptortus pedestris

\section{Introduction}

Symbiotic microorganisms are common in insects (Buchner, 1965). They are usually transmitted vertically from mother to offspring and occupy unique ecological niches, such as midgut crypts and specialized tissues and cells, within their host insects (Baumann and Moran, 1997). Symbiotic microbes participate in the biology of their hosts in a variety of ways, for example, provisioning of

Correspondence: T Fukatsu, Bioproduction Research Institute, National Institute of Advanced Industrial Science and Technology (AIST), Tsukuba 305-8566, Japan.

E-mail: t-fukatsu@aist.go.jp

or BL Lee, Global Research Laboratory, College of Pharmacy, Pusan National University, Jang-Jeong Dong, Kum-Jeong Ku, Pusan 609735, South Korea.

E-mail: brlee@pusan.ac.kr

Received 13 June 2013; revised 27 August 2013; accepted 28 August 2013; published online 3 October 2013 essential nutrients (Moran et al., 2008), defense against natural enemies and adaptation to specific ecological conditions (Oliver et al., 2010) and promoting negative fitness effects and reproductive aberrations (Werren et al., 2008).

The infection density of a symbiont within its host is among the most critical factors for shaping the biological effect of the symbiotic association. Previous studies have shown that the infection density of the symbiont can affect the fidelity of vertical transmission, the degree of reproductive aberrations and the level of fitness consequences as indicated by body size, growth, survival and/or fecundity (McGraw et al., 2002; Koga et al., 2003; Mouton et al., 2004; Sakurai et al., 2005; Kikuchi et al., 2007; Kim et al., 2013b). The mechanisms underlying the infection, proliferation and maintenance of symbionts within their host organisms are therefore of ecological and evolutionary importance (Kondo et al., 2005; Engelstadter et al., 2006; Vautrin and 
Vavre, 2009). However, the fastidious nature and consequent genetic intractability of insect symbionts have been major obstacles in understanding the mechanisms of their infection dynamics (Pontes and Dale, 2006). Previous studies on symbiont infection density have been conducted on whole insects without symbiont cultivation: different combinations of host and symbiont genotypes were experimentally generated by microinjection, antibiotic therapy and/or introgression, and infection densities of the symbionts in the different host-symbiont combinations were evaluated by quantitative PCR. These studies have demonstrated that symbiont infection dynamics are significantly affected by the symbiont genotype (McGraw et al., 2002; Ikeda et al., 2003; Mouton et al., 2003, 2004), host genotype (Kondo et al., 2005; Mouton et al., 2007), interactions between co-infecting symbionts (Koga et al., 2003; Kondo et al., 2005; Sakurai et al., 2005; Goto et al., 2006) and/or other environmental factors (Mouton et al., 2007; Anbutsu et al., 2008). However, it is not clear which symbiont genes are involved in the infection dynamics and regulation of symbiont density.

Apart from the insect-microbe symbioses, there are several model systems in which beneficial symbiotic bacteria have been cultured and genetically manipulated to understand the molecular crosstalk between symbiont and host. In such model systems as squid-Vibrio, legume-Rhizobium and nematode-Photorhabdus symbioses (Gage, 2004; Nyholm and McFall-Ngai, 2004; Goodrich-Blair and Clarke, 2007), a number of symbiont genes have been identified to be important for the symbiotic associations. These genes have been classified into three categories based on their mutant phenotypes in symbiosis, which include (i) initiation mutants, which are unable to establish infection in the host, (ii) accommodation mutants, which can establish infection but cannot reach normal infection density and (iii) persistence mutants, which establish infection normally but are unable to maintain the normal infection level (Ruby, 2008). Such experimental model systems have not been available for insect symbiosis studies.

Recently, the bean bug, Riptortus pedestris (Hemiptera: Alydidae), has been recognized as an ideal insect model for investigating insect-bacterium symbiosis. $R$. pedestris harbors a beneficial and specific bacterium in a specialized region of the posterior midgut (Kikuchi et al., 2005). This symbiont belongs to a member of the betaproteobacterial genus Burkholderia, and is acquired orally by host nymphs from the environment every generation. Further, Burkholderia can be easily cultivable and genetically manipulatable (Kikuchi et al., 2007, 2011a, b; Kikuchi and Fukatsu, 2013; Kim et al., 2013a, b). Using this model system, we performed transposon-mediated random mutagenesis of Burkholderia symbiont strain and screened the mutant strains for their symbiotic capabilities in the Riptortus host. Among $>1800$ mutant strains, we identified a symbiosis-deficient mutant strain in which purL, a gene involved in the purine biosynthesis pathway, was disrupted by a transposon insertion. The purL mutant of the Burkholderia symbiont was investigated in detail for its microbiologic properties, infection density dynamics and effects on the host insect. Furthermore, a strain containing a mutation in another purine biosynthesis gene, purM, was generated to confirm whether the phenotypes of the purL mutant are due to the disruption of purine biosynthesis.

\section{Materials and methods}

Bacteria and media

Escherichia coli cells were cultured at $37^{\circ} \mathrm{C}$ in $\mathrm{LB}$ medium ( $1 \%$ tryptone, $0.5 \%$ yeast extract, $0.5 \%$ $\mathrm{NaCl})$. Burkholderia symbiont RPE75 cells were cultured at $30{ }^{\circ} \mathrm{C}$ in YG medium $(0.4 \%$ glucose, $0.5 \%$ yeast extract, $0.1 \% \mathrm{NaCl}$ ) (Kikuchi et al., 2011b). The following supplements were added to the culture media unless otherwise indicated: $30 \mu \mathrm{g} \mathrm{ml}^{-1}$ rifampicin, $30 \mu \mathrm{g} \mathrm{ml}^{-1} \mathrm{kanamycin}$ and/or $300 \mu \mathrm{g} \mathrm{ml}^{-1}$ 2,6-diaminopimelic acid (DAP).

Generation of mutants

Methods for transposon mutagenesis, homologous recombination and generation of complemented mutant strains are described in the Supplementary Information.

\section{Insect rearing and symbiont inoculation}

$R$. pedestris was maintained in our insect laboratory at $26^{\circ} \mathrm{C}$ under a long day cycle of $16 \mathrm{~h}$ light and $8 \mathrm{~h}$ dark as described (Kim et al., 2013a). Nymphal insects were reared in clean plastic containers with soybean seeds and distilled water containing $0.05 \%$ ascorbic acid (DWA). When newborn nymphs molted to the second instar stage, symbiont inoculum solution was provided using wet cotton balls in a small Petri dish. The inoculum solution consisted of mid-log phase Burkholderia cells in DWA at a concentration of $10^{7}$ cells per ml. For inoculation of the mutant strain, higher inoculum concentrations $\left(10^{8}\right.$ and $10^{9}$ cells per $\left.\mathrm{ml}\right)$ were also used. To generate insects without the Burkholderia symbiont, a group of second instar nymphs were reared in a separate clean container and provided with only sterilized DWA and soybeans.

Measurement of bacterial growth in liquid media Growth curves of the Burkholderia symbiont strains were examined in either YG medium or minimal medium $\left(0.2 \%\right.$ glucose, $0.2 \%\left(\mathrm{NH}_{4}\right)_{2} \mathrm{SO}_{4}, 1.36 \%$ $\mathrm{KH}_{2} \mathrm{PO}_{4}, \quad 0.00005 \% \quad \mathrm{FeSO}_{4} \cdot 7 \mathrm{H}_{2} \mathrm{O}, 1 \mathrm{mM} \quad \mathrm{MgSO}_{4}$ ). The starting cell solutions were prepared from stationary phase cells by adjusting $\mathrm{OD}_{600}$ to 0.05 in 
either YG medium or minimal medium. The culture media were incubated on a rotator shaker at 180 r.p.m. at $30^{\circ} \mathrm{C}$ for $36 \mathrm{~h}$ and $\mathrm{OD}_{600}$ was monitored every $3 \mathrm{~h}$ using a spectrophotometer (Mecasys, Daejeon, Korea). One of the following purine derivatives was added to the culture medium at a final concentration of $0.5 \mathrm{~mm}$ : adenine, adenosine, adenosine monophosphate (AMP), inosine monophosphate (IMP) or 5-amino-1- $\beta$-D-ribofuranosyl-imidazole-4-carboxamide (AICAR) (see Supplementary Figure S1).

\section{Analysis of biofilm formation}

Mid-log phase Burkholderia symbiont cells were adjusted $\mathrm{OD}_{600}$ to 0.8 in YG medium and $150 \mu \mathrm{l}$ of the cell suspension was added to each well of a 96-well plate for each sample. To examine the effects of purine derivatives in rescuing the mutant defect, adenine, adenosine, AMP, IMP or AICAR was added to each well at a final concentration of $0.5 \mathrm{~mm}$. The 96-well plates were incubated at $30^{\circ} \mathrm{C}$ for $48 \mathrm{~h}$ with shaking at 110 r.p.m. At the end of the incubation, the wells were washed three times with phosphate-buffered saline (PBS: $8 \% \mathrm{NaCl}, 1.16 \%$ $\mathrm{Na}_{2} \mathrm{HPO}_{4} \cdot 7 \mathrm{H}_{2} \mathrm{O}, \quad 0.2 \% \quad \mathrm{KH}_{2} \mathrm{PO}_{4}, 0.2 \% \mathrm{KCl}$ ) and adherent biofilms were fixed with $99 \%$ methanol for $10 \mathrm{~min}$. After removing the methanol and airdrying the samples, each well was incubated with $200 \mu \mathrm{l}$ of $0.1 \%$ crystal violet in methanol for $10 \mathrm{~min}$, washed in running tap water and air-dried. The stained dye was then solubilized by adding $200 \mu \mathrm{l}$ of $30 \%$ acetic acid to each well and $\mathrm{OD}_{540}$ was measured using a plate reader (Multiskan EX; Thermo Scientific).

\section{Electrophoretic analysis of bacterial carbohydrates} Bacterial cells were suspended in sample buffer (60 mм Tris-HCl, pH 6.8, 2\% SDS, 10\% glycerol, $0.005 \%$ bromophenol blue), boiled at $95{ }^{\circ} \mathrm{C}$ for $5 \mathrm{~min}$, incubated with $400 \mu \mathrm{g} \mathrm{ml}^{-1}$ proteinase $\mathrm{K}$ at $60{ }^{\circ} \mathrm{C}$ for $1 \mathrm{~h}$ and boiled again prior to electrophoresis. The amount of sample for loading was adjusted to $1 \times 10^{8}$ cells per lane for conventional SDS-PAGE (Laemmli, 1970) in $12 \%$ gels and $2 \times 10^{8}$ cells per lane for Tricine SDS-PAGE (Schägger, 2006) in 12\% gels. Bacterial carbohydrates separated in the gels were visualized using the Pro-Q Emerald 300 Lipopolysaccharide Gel Stain Kit (Invitrogen, Carlsbad, CA, USA).

\section{Colony-forming unit assay}

The midgut fourth (M4) region was dissected out of $R$. pedestris, placed in $100 \mu \mathrm{l}$ of $10 \mathrm{~mm}$ phosphate buffer (PB, pH 7.0), homogenized by a plastic pestle and serially diluted with $\mathrm{PB}$. The diluted samples were spread on rifampicin-containing YG agar plates. After 2 days of incubation at $30^{\circ} \mathrm{C}$, colonies on the plates were counted and colony-forming units (CFUs) per insect were calculated by multiplying the colony counts by the dilution factor.

\section{Quantitative PCR}

Dissected midgut M3 and M4 regions were homogenized and DNA was extracted using QIAamp DNA mini kit (Qiagen, Hilden, Germany). The DNA samples were analyzed by quantitative PCR targeting dnaA gene of the Burkholderia symbiont. Real-time quantitative PCR was performed using QuantiMix SYBR Kit (PhileKorea, Daejeon, Korea) and CFX96 Real-time system (BioRad, Hercules, CA, USA) with primers BSdnaA-F (5'-AGCGCGAGATCAGACGGTCG TCGAT- $3^{\prime}$ ) and BSdnaA-R (5'-TCCGGCAAGTCGCGC ACGC-3') as previously described (Kim et al., 2013a).

\section{Microscopic observation of midgut crypts}

To observe the midgut morphology, $R$. pedestris insects were sterilized with $70 \%$ ethanol and dissected under a dissection microscope (Olympus SZ21) in a glass Petri dish filled with PBS. For dissection microscopy, pictures were taken by a digital camera attached to the microscope and processed using the iSolution Lite program (Olympus, Tokyo, Japan). For fluorescence microscopy, dissected midgut M4 regions of $R$. pedestris were fixed with $4 \%$ paraformaldehyde in PBS for $10 \mathrm{~min}$ at room temperature, washed twice with PBS, incubated in PBS containing $0.1 \%$ Triton X-100 for $5 \mathrm{~min}$ and stained with $0.5 \mu \mathrm{M}$ SYTOX Green (Molecular Probes, Eugene, OR, USA) and $5 \mathrm{Uml}^{-1}$ Alexa Fluor 568 phalloidin (Molecular Probes) in PBS for $20 \mathrm{~min}$. After washing twice with PBS, the samples were mounted on silane-coated glass slides and examined under a laser-scanning confocal microscope (TCS SP2 ABOS; Leica, Solms, Germany), as previously described (Kikuchi et al., 2011b). For transmission electron microscopy, dissected midgut M4 regions were prefixed with $2.5 \%$ glutaraldehyde in $0.1 \mathrm{M}$ sodium cacodylate buffer, $\mathrm{pH} 7.4$ (SCB), at $4{ }^{\circ} \mathrm{C}$ for $18 \mathrm{~h}$. The samples were washed three times with $0.1 \mathrm{M} \mathrm{SCB}$ at room temperature for $15 \mathrm{~min}$ each and postfixed with $1 \%$ osmium tetroxide in $0.1 \mathrm{M}$ SCB for $1 \mathrm{~h}$ at room temperature. After three times washing, the samples were dehydrated and cleared through an ethanol and propylene oxide series and embedded in Epon 812 resin. The embedded samples were trimmed and sectioned on an ultramicrotome (Reichert SuperNova, Leica), stained with uranyl acetate and lead citrate and observed under a transmission electron microscope (HITACHI H-7600, Hitachi, Tokyo, Japan).

\section{Fitness measurement}

At the middle age of third-, fourth- and fifth-instar stages, insects were examined for their body length and dry body weight. The body length was measured from the head to the tip of the abdomen. 
For dry weight measurement, the insects were immersed in acetone for $5 \mathrm{~min}$ and then completely dried by incubation in a $70^{\circ} \mathrm{C}$ oven.

\section{Results}

A transposon-inserted Burkholderia mutant induced a less-developed host symbiotic organ

To screen symbiosis-deficient mutants of the Burkholderia symbiont, we performed random Tn5 transposon mutagenesis on the symbiont strain RPE75. In total, 1860 transposon-inserted Burkholderia clones were isolated and orally administered to early second-instar nymphs of $R$. pedestris. When these insects reached the third-instar stage, their midgut M4 region was dissected, morphologically examined and subjected to PCR detection of symbiotic bacteria. While most of the mutant-infected insects exhibited normal midgut phenotypes that were indistinguishable from those of the wildtypeinfected insects (Figure 1a), we found an interesting mutant in which the host exhibited less-developed midgut crypts. The crypts of the insects infected with the mutant were smaller and less hazy than those of the insects infected with the wildtype symbiont (Figure 1b). The PCR results showed that the crypts were certainly infected with the Burkholderia mutant (Figure 1c), but the CFU assay of the dissected midgut M4 regions revealed significantly lower infection densities of the Burkholderia mutant than those of the wildtype strain (Figure 1d).

Purine synthesis gene purL was disrupted in the Burkholderia mutant

Using a plasmid rescue procedure, we cloned and sequenced the transposon insertion site of the mutant genome and identified a disruption in the purL gene (Figure 1e). The purL gene encodes $\mathrm{N}$-formylglycinamidine ribonucleotide (FGAM) synthetase, which is involved in the fourth step of bacterial purine biosynthesis (Supplementary Figure S1). When the wildtype and purL mutant strains were cultured in nutritionally rich YG medium, both Burkholderia strains initially grew well, but the purL mutant exhibited lower (approximately a half) bacterial titers than the wildtype strain at the stationary phase (Figure 2a). When adenine or adenosine was added to the culture

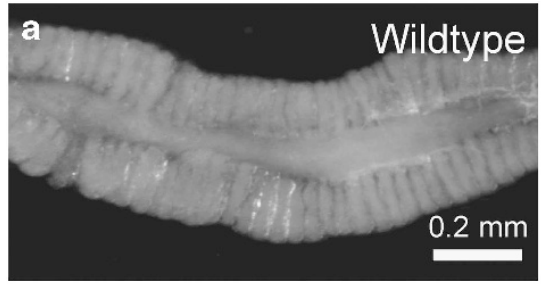

c
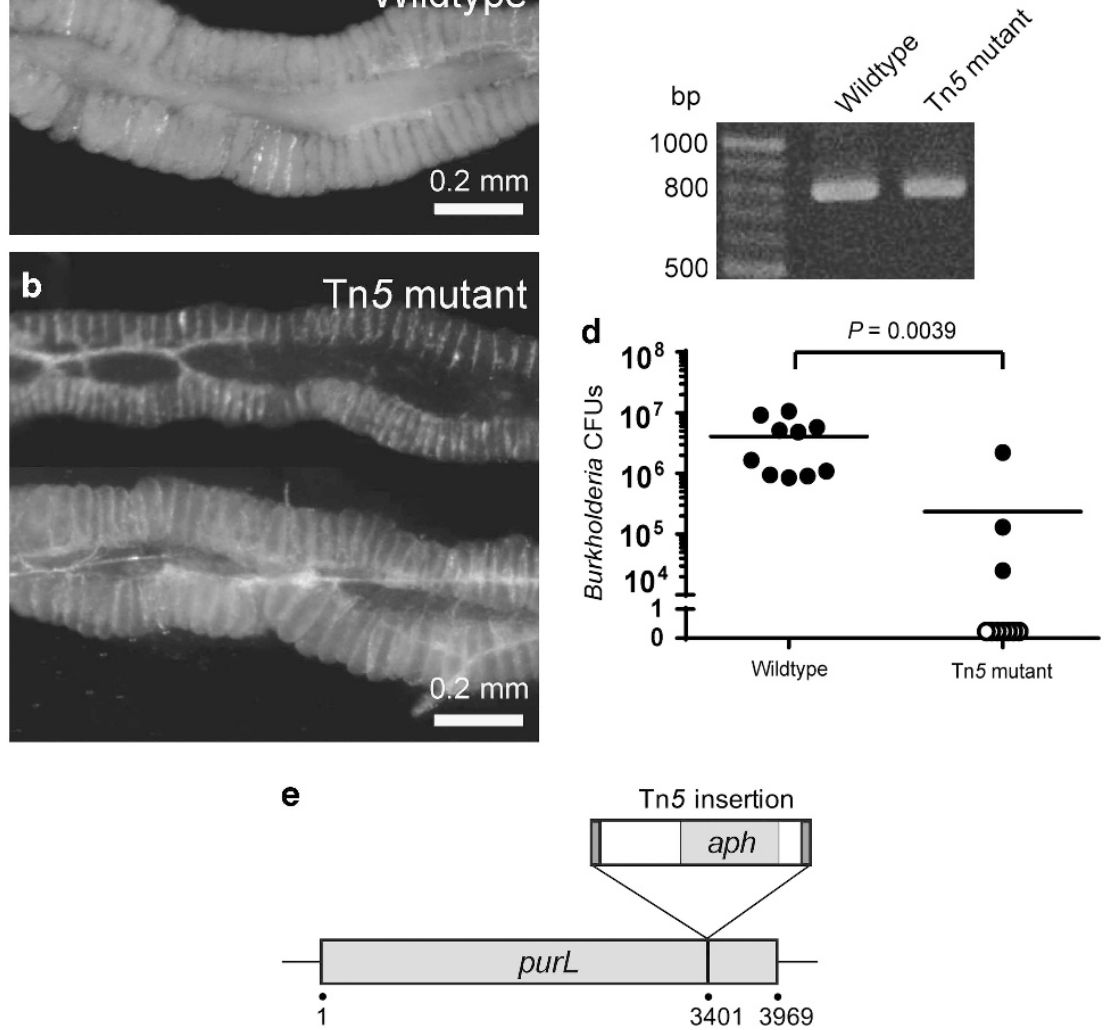

Figure 1 (a, b) Morphological examination of the midgut symbiotic organ of R. pedestris: a M4 region dissected from wildtype-infected third-instar nymph (a) and two M4 regions dissected from Tn5 mutant-infected third-instar nymphs (b). (c) PCR detection of Burkholderia symbiont in M4 regions dissected from wildtype-infected and Tn5 mutant-infected third-instar nymphs. (d) Infection densities of Burkholderia symbiont in M4 regions dissected from wildtype-infected and Tn5 mutant-infected third-instar nymphs ( $n=10$, respectively) evaluated by CFU assay. P-value of unpaired $t$-test is shown on the graph. (e) The purL gene of the Burkholderia mutant disrupted by an insertion of Tn 5 transposon, which contains a kanamycin resistant gene (aph) and inverted repeats at both ends, at the site 3401 . 

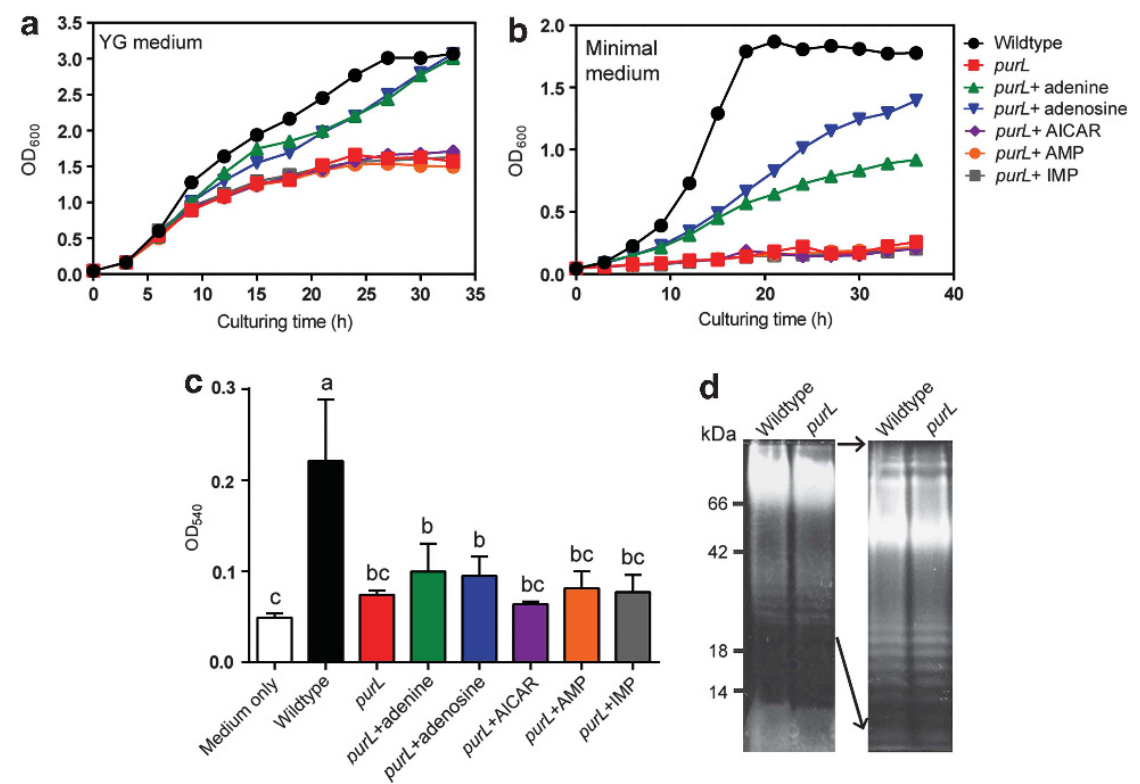

Figure 2 (a, b) Growth curves of the wildtype and purL mutant strains of Burkholderia symbiont under cultured conditions in YG medium (a) and minimal medium (b). Purine derivatives (adenine, adenosine, AICAR, AMP or IMP) were added to the media to examine the auxotrophic phenotype of the purL mutant. (c) Quantitative comparison of biofilm formation by the wildtype and purL mutant strains of Burkholderia symbiont cultured in YG medium. Different letters (a-c) on the top of the columns indicate statistically significant differences $(P<0.05$; one-way ANOVA with Tukey's correction). (d) Lipopolysaccharide profiles of the wildtype and purL mutant strains of Burkholderia symbiont cultured in YG medium. Left and right panels show Tricine SDS-PAGE and conventional SDS-PAGE, respectively.

medium, the growth of the purL mutant was markedly improved, whereas addition of AICAR, AMP or IMP had little effects on the growth of the mutant (Figure 2a). When cultured in nutritionally restricted minimal medium, the purL mutant exhibited little growth, whereas adenine and adenosine, but not AICAR, AMP or IMP, remarkably restored the growth of the purL mutant (Figure 2b). These results indicate that the transposon insertion in the mutant genome disrupts the purL gene not only structurally but also functionally.

Attenuated biofilm formation in the purL mutant was not restored by purine supplementation

As a recent study on the purL mutant of Photorhabdus, symbiont of nematode, showed an inability to form biofilm in vitro and a partial recovery of biofilm formation by addition of AICAR (An and Grewal, 2010), the wildtype and purL mutant strains of the Burkholderia symbiont were tested for their ability to form biofilm. The purL mutant strain exhibited significantly lower biofilm formation than the wildtype strain (Figure 2c). Even when adenine, adenosine and other purine derivatives were added to the culture medium, the biofilm production of the purL mutant was not improved (Figure 2c). When we analyzed the lipopolysaccharide patterns on SDS-PAGE gels, no apparent qualitative or quantitative differences were observed between the wildtype and purL mutant strains (Figure 2d). These in vitro results indicate that (i) the purL mutant exhibits attenuated biofilm production, (ii) the attenuated biofilm production cannot be restored by supplementation of purine derivatives and (iii) the inability of the purL mutant to form biofilm may be independent of the growth defect and unrelated to the lipopolysaccharide-synthesizing capability.

The purL mutant exhibited low infection efficiency to the host symbiotic organ, whereas higher inoculum titers improved the efficiency

To examine the infection efficiency of the purL mutant, early second instar nymphs of $R$. pedestris were orally administered with the wildtype or purL mutant at a concentration of $10^{7}$ bacterial cells per ml. The midgut M4 regions of the insects were removed at $24 \mathrm{~h}$ post-inoculation and subjected to CFU assays (Figure 3). Of 20 insects inoculated with the wildtype strain, 16 insects $(80 \%)$ were infected with the symbiont and the infection densities were $\sim 5.5 \times 10^{3}$ bacterial cells per insect. By contrast, of 20 insects inoculated with the purL mutant, only 6 insects $(30 \%)$ were infected and the infection densities were on average $<10$ bacterial cells per insect. When the inoculum titers of the purL mutant were increased to $10^{8}$ and $10^{9}$ bacterial cells per ml, both the infection rates and densities were improved to levels closer to those of the wild-type strain (Figure 3). These results indicate that (i) the purL mutant is significantly less efficient to establish infection in the host symbiotic organ than the wildtype strain and (ii) the inferior infection 


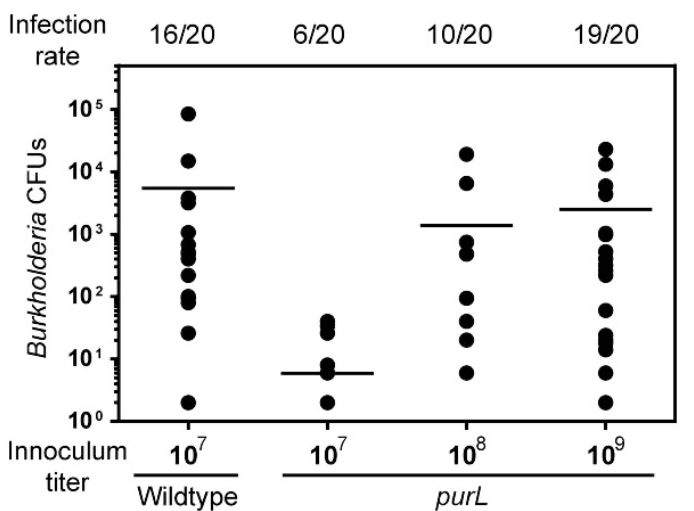

Figure 3 Infection rates and densities of the wildtype and purL mutant strains of Burkholderia symbiont following inoculation to second-instar nymphs of $R$. pedestris. Symbiont titers in dissected M4 regions were measured by CFU assay at $24 \mathrm{~h}$ post-inoculation of wildtype $\left(10^{7}\right.$ cells per ml) or purL mutant $\left(10^{7}-10^{9}\right.$ cells per ml). A total of 20 insects per group were examined. Infection rates and inoculum titers are indicated at the top and bottom of the graph, respectively. Horizontal lines in the graph indicate mean values.

efficiency of the purL mutant can be compensated by higher bacterial inoculum titers. Based on these results, the higher inoculum titer $\left(10^{9}\right.$ bacterial cells per $\mathrm{ml}$ ) of the purL mutant was used in in vivo symbiotic characterization of host gut development and fitness to ensure proper infection with the purL mutant.

Symbiotic accommodation defect of the purL mutant was observed during the initial infection process

To understand the low infection efficiency of the purL mutant, we further characterized the initial infection process after inoculation of either the wildtype or purL mutant strain. The copy number of the Burkholderia dnaA gene was examined by quantitative PCR for midgut samples dissected from second-instar nymphs at $6,9,12,15,18,21$ and $24 \mathrm{~h}$ post-infection (Figure 4). At $6 \mathrm{~h}$ post-infection, both the wildtype and purL mutant strains were detected at similarly low levels in the midgut M3 and M4 regions (10-30 dnaA copies in M3 and $\leq 10$ in M4). However, at later time points, the wildtype and purL mutant strains exhibited strikingly different infection dynamics. The titer of the wildtype strain increased exponentially in M4 and ultimately attained infection titers over $10^{4} d n a A$ gene copies. By contrast, the titer of the purL mutant strain increased slowly and attained infection titers around $10^{2} d n a A$ gene copies (Figure 4). These results strongly suggest that the purL mutant is initially able to colonize the midgut M4 region but subsequently fails to maintain normal infection levels in the host symbiotic organ.

The purL mutant-infected insects exhibited attenuated development of the host symbiotic organ

As the purL mutant was initially identified by the observation of a less-developed symbiotic organ, the size and morphology of the midgut M4 region of

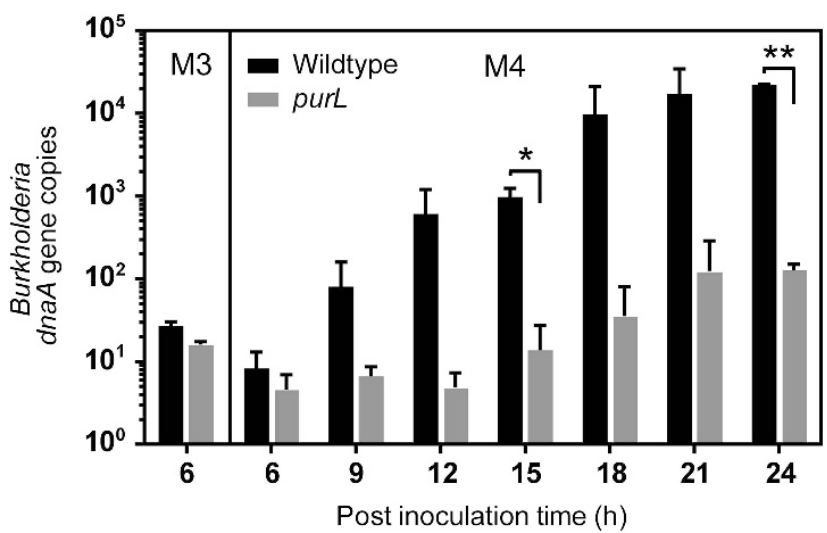

Figure 4 Initial infection dynamics of the wildtype and purL mutant strains of Burkholderia symbiont following inoculation to second instar nymphs of $R$. pedestris. The insects were orally administrated with standard infection solution containing wildtype or purL mutant $\left(10^{7}\right.$ cells per $\left.\mathrm{ml}\right)$, their M4 regions of the midgut were dissected at $6-24 \mathrm{~h}$ post-inoculation and 20 midguts per sample were combined and subjected to DNA extraction and quantitative PCR of symbiont dnaA gene copies. At $6 \mathrm{~h}$ postinfection, M3 samples were also subjected the quantitative PCR analysis. Two independent experiments were performed. Asterisks indicate statistically significant differences (unpaired $t$-test; ${ }^{*} P=0.03,{ }^{*} P=0.0003$ )

second instar nymphs were examined at $12,24,36$, 48 and $60 \mathrm{~h}$ post-inoculation in uninfected, wildtype-infected and purL mutant-infected insects (Figure 5a). Throughout the time course, the wildtype-infected M4 region steadily increased in width up to an average of $0.26 \mathrm{~mm}$ at $60 \mathrm{~h}$, whereas the uninfected M4 region showed only slight growth to $0.13 \mathrm{~mm}$ in width. The purL mutant-infected M4 region exhibited an intermediate growth at $0.20 \mathrm{~mm}$ in width (Figure 5a). Confocal microscopic images of the midgut M4 crypts at 24 and $48 \mathrm{~h}$ after inoculation histologically confirmed these patterns as follows: well-developed midgut crypts were densely colonized with the wildtype strain (Figures $5 \mathrm{~b}$ and e); much less-developed midgut crypts were sparsely colonized with the purL mutant (Figures 5c and f) and virtually no midgut crypts developed without symbiont infection (Figures $5 \mathrm{~d}$ and g). These results demonstrate that (i) the midgut crypts infected with the purL mutant are significantly less developed than those infected with the wildtype strain and that (ii) the lower infection density of the purL mutant may be responsible for the attenuated development of the host symbiotic organ.

Adenine and adenosine were unable to rescue the symbiotic phenotype induced by the purL mutant in vivo

In the in vitro characterization of the purL mutant, the growth defect was rescued by supplying adenine and adenosine to the medium (Figure 2). To assess whether adenine and adenosine could rescue the accommodation defect in vivo, uninfected, wildtype-infected and purL mutant-infected insects were 

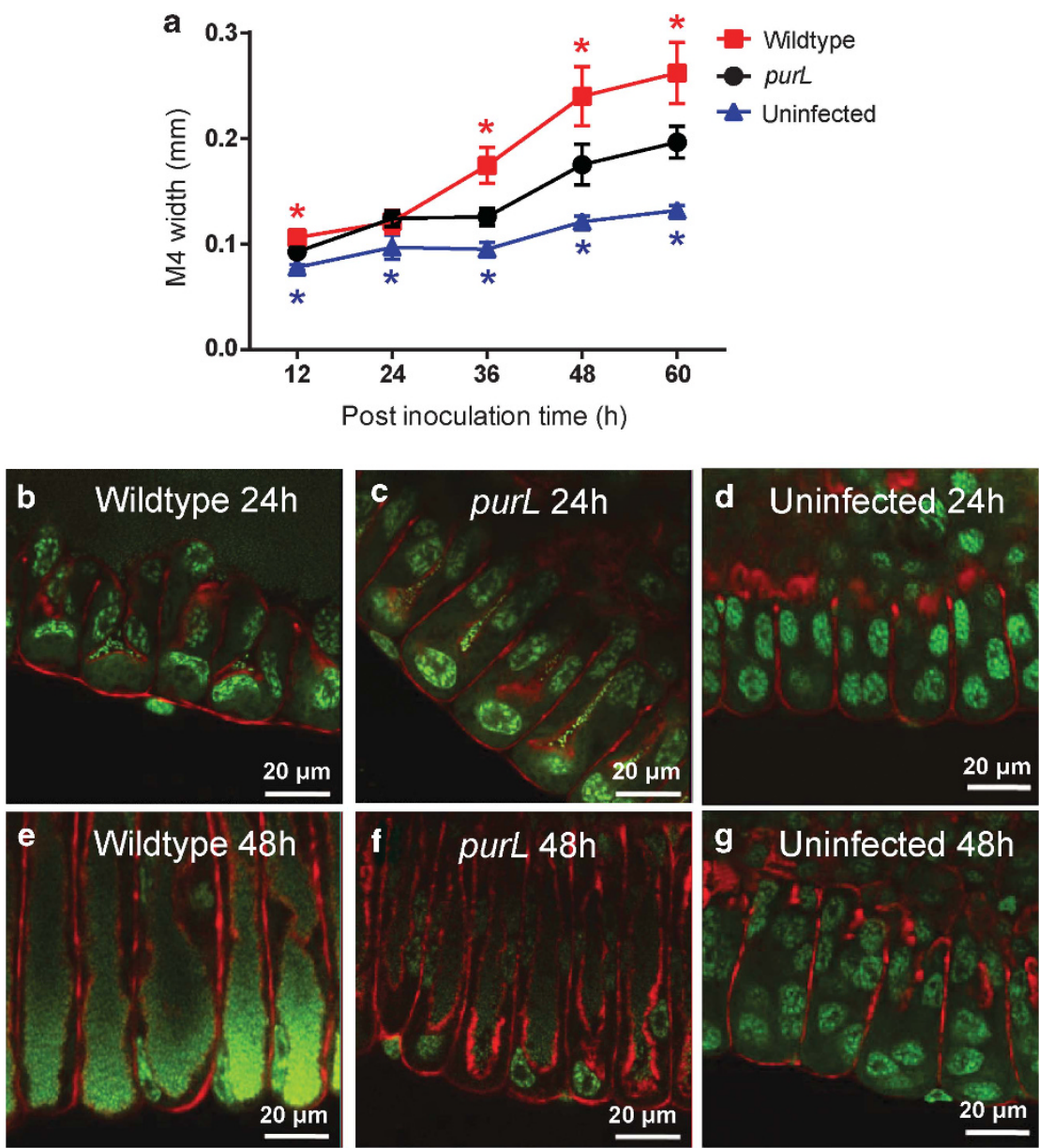

Figure 5 (a) Development of the midgut symbiotic organ after inoculation of the wildtype and purL mutant strains of Burkholderia symbiont to second-instar nymphs of $R$. pedestris. The insects were dissected at 12, 24, 36, 48 and $60 \mathrm{~h}$ post-inoculation and the width of their M4 region was measured. Uninfected insects were also examined in the same way. Mean and standard deviation of five measurements are shown for each time point. Asterisks indicate statistically significant differences between wildtype-infected insects and purL mutant-infected insects (red) and those between purL mutant-infected insects and uninfected insects (blue) (unpaired $t$-test; $P<0.05)$. (b-g) Confocal microscopic images of the midgut crypts in the wildtype-infected insects (b, e), purL mutant-infected insects $(\mathbf{c}, \mathbf{f})$ and uninfected insects $(\mathbf{d}, \mathbf{g})$ at $24 \mathrm{~h}$ and $48 \mathrm{~h}$ post-inoculation. Green signals visualize nuclear DNA of crypt cells, whereas red signals indicate actin fibers underlining crypt cell membrane. In wildytpe-infected insects (b, e) and purL-infected insects (c, f), green signals also depict Burkholderia symbiont DNA within crypt cavities.

continuously fed with water containing $0.5 \mathrm{~mm}$ adenine or adenosine. However, the in vivo supplementation of adenine and adenosine did not increase the early infection densities of the purL mutant (Supplementary Figure S2a). Furthermore, neither adenine nor adenosine was able to restore crypt development (Supplementary Figure S2b-h). These data suggest that an insufficient amount of adenosine reached the symbiotic organ or that the in vivo phenotype induced by the purL mutant infection was not solely due to the growth defect of the purL mutant.

Lower infection density and less-developed symbiotic organ were observed in the purL mutant-infected insects throughout the nymphal stages

To address the effects of the purL mutant infection on older insects, we monitored the morphology of the midgut M4 region in third-, fourth- and fifth-instar nymphs that were infected with either the wildtype or purL mutant strain. Although the size of the crypts was similar between the purL mutant-infected insects and the wildtype-infected insects, the coloration of the crypts was conspicuously less hazy (indicative of lower symbiont density) in the purL mutant-infected insects than the wild-type-infected insects (Figures 6a-f). CFU assays of the midgut M4 regions confirmed the morphological observations: the symbiont titers in the purL mutant-infected insects were consistently and significantly lower than those in the wildtypeinfected insects (Figure 6g). These results indicate that the lower infection density of the purL mutant is not restricted to the early second-instar stage but is observed throughout the lifetime of the Riptortus host. Transmission electron microscopic images of the midgut M4 crypts of third-instar nymphs revealed that the large, coccal and often degenerative purL 

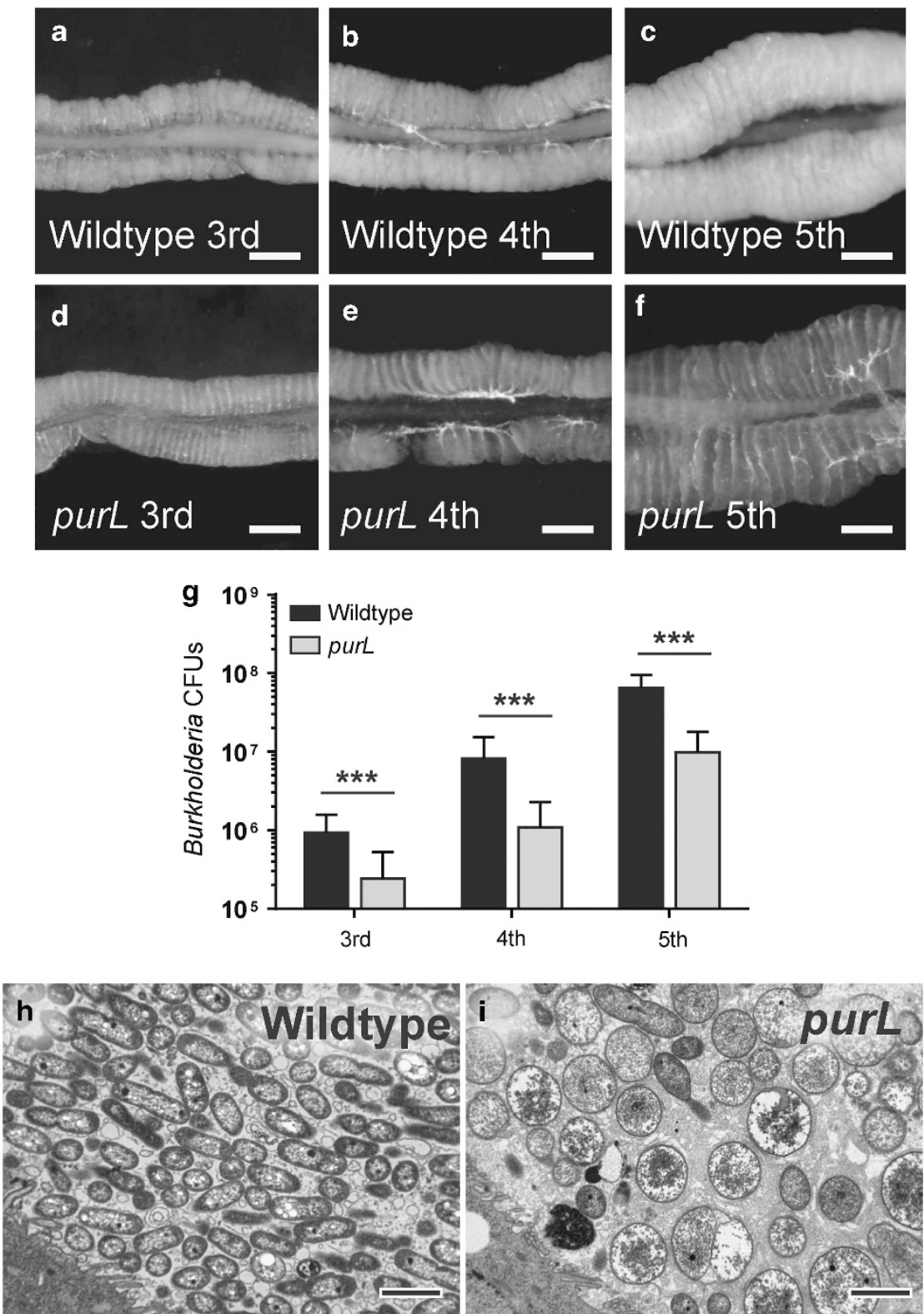

Figure 6 (a-f) Light microscopic images of the midgut symbiotic organ dissected from third- (a, d), fourth- (b, e) and fifth- (c, f) instar nymphs of $R$. pedestris infected with the wildtype $(\mathbf{a}-\mathbf{- c})$ and purL mutant $(\mathbf{d}-\mathbf{f})$ strains of Burkholderia symbiont. Scale bars show $0.2 \mathrm{~mm}$. (g) Infection titers of the wildtype and purL mutant strains of Burkholderia symbiont in M4 region of the midgut symbiotic organ. Means and standard deviations $(n=20)$ are shown as columns and error bars, respectively. Asterisks indicate statistically significant differences (unpaired $t$-test; $\left.{ }^{* * *} P<0.0001\right)$. (h, i) Transmission electron microscopic images of midgut crypts of third-instar nymphs infected with wildtype strain (h) and purL mutant (i) of Burkholderia symbiont. Scale bars show $2 \mu \mathrm{m}$.

mutant cells were morphologically distinct from the rod-shaped wildtype cells (Figures $6 \mathrm{i}$ and h), suggesting a dramatic difference in symbiotic accommodation between the wildtype and purL mutant strains.

The purL mutant-infected host insects exhibited attenuated fitness

To determine the effects of the purL mutant on host fitness, we measured the body size and weight of third-, fourth- and fifth-instar nymphs infected with either the wildtype or purL mutant strain. Both the body lengths and dry weights of the purL mutantinfected insects were consistently and significantly smaller than those of the wildtype-infected insects (Figures 7a and b). These results indicate that the Riptortus host infected with the purL mutant exhibits attenuated fitness compared with the host infected with the wildtype strain.

The purM deletion mutant showed impaired growth and biofilm formation in vitro

To rule out the possibility that secondary mutations in the purL transposon-induced mutant contribute to the purL phenotypes, we targeted another purine biosynthesis gene, purM, to construct a 

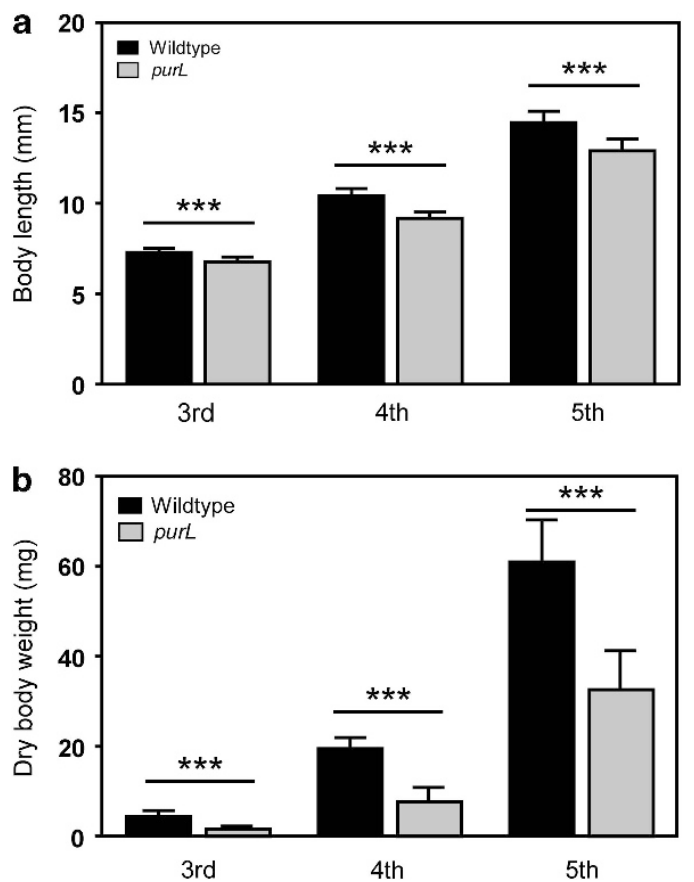

Figure 7 Fitness values of $R$. pedestris infected with wildtype strain and purL mutant of Burkholderia symbiont. Means and standard deviations $(n=50)$ of body length (a) and dry body weight (b) of third-, fourth- and fifth-instar nymphs are shown. Asterisks indicate statistically significant differences (unpaired t-test; $\left.{ }^{* * *} P<0.0001\right)$

deletion mutant. PurM is a $5^{\prime}$-aminoimidazole ribonucleotide synthetase that uses the product of PurL, FGAM, to synthesize $5^{\prime}$-aminoimidazole ribonucleotide (Supplementary Figure S1). In addition to the $\Delta$ purM mutant strain, we also generated a complemented strain ( $\Delta$ purM/purM) by transforming the $\Delta$ purM mutant with a plasmid encoding a functional purM gene. The in vitro growth rates of the $\Delta$ purM mutant were similar to those of the purL mutant (see Figures 2a and b), exhibiting slower growth in YG medium than the wildtype strain (Figure 8a) and little growth in minimal medium (Figure 8b). By adding adenosine to the media, the growth of the $\Delta$ purM mutant was partially restored. When the $\Delta$ purM mutant was complemented with the functional purM gene ( $\Delta$ purM/purM), the growth rates were fully restored in both media (Figures $8 \mathrm{a}$ and b). Biofilm formation of the $\Delta$ purM mutant was also similar to that of the purL mutant: the $\Delta p u r M$ mutant produced much lower amounts of biofilm than the wildtype strain, and supplementation of adenosine did not improve biofilm formation (Figure 8c). On the other hand, the $\Delta$ purM/purMcomplemented mutant exhibited biofilm formation to the level observed in the wildtype stain (Figure 8c).

The purM deletion mutant exhibited the same in vivo phenotypes as the purL mutant

To determine whether the symbiotic properties of the $\Delta$ purM mutant were similar to those of

the purL mutant, their infection densities in third-, fourth- and fifth-instar nymphs were determined by CFU assay. The symbiont titers of the $\Delta$ purM-infected insects were significantly lower than those of the wildtype-infected insects, and the titers were restored in the $\Delta$ purM/purM-infected insects (Figure 8d). The morphological observations of the midgut M4 regions of fifth-instar nymphs also reflected the results of the symbiont titers (Figure 8e): the M4 crypts of the $\Delta p u r M$-infected insects were much less hazy than those of the wildtype- and $\Delta$ purM/purM-infected insects. The lower infection density of the $\Delta$ purM mutant negatively affected the fitness values of the host insects: the body lengths and dry weights of the $\Delta$ purM mutantinfected insects were significantly smaller than those of the wildtype-infected insects, whereas the fitness values of the $\Delta$ purM/purM-infected insects were similar to those of the wildtype-infected insects (Figures $8 \mathrm{f}$ and $\mathrm{g}$ ). These in vitro and in vivo results with the $\Delta$ purM mutant strongly suggest that the phenotypes of the purL mutant are caused by the disruption of the purL gene and not by secondary mutations occurring elsewhere in the symbiont genome during transposon mutagenesis.

\section{Discussion}

In this study, we demonstrate that the purine biosynthesis mutants, purL and purM, of the Burkholderia symbiont are able to colonize the host symbiotic organ but are incapable of accommodation and consequently fail to establish normal symbiotic association with the host. The host insects infected with these mutants exhibited significantly smaller body size than the host insects infected with the wildtype strain of the Burkholderia symbiont, which is likely due to the lower infection density of the symbiont mutants in the host midgut compared with the wildtype strain.

Why does the purL mutant fail to attain normal infection density in the Riptortus host?

The purL mutant exhibited a low level of initial colonization and overall low population in the midgut M4 region in vivo. The clues to the reason why the purL mutant exhibits these in vivo properties may be found in its in vitro phenotypes. First, the auxotrophic phenotype of the purL mutant showed decreased growth in nutrient rich medium and almost no growth in minimal medium (Figures 2a and b). Our recent study showed that polyhydroxyalkanoate biopolymer, which supports bacterial survival in hostile environments, is required for the Burkholderia symbiont to persist in the host midgut, suggesting that the symbiotic environment may be restrictive and/or hostile to the symbiont (Kim et al., 2013b). Although we do not know the exact nutritional environment of the midgut M4 region, we suspect that M4 may have limited purine 

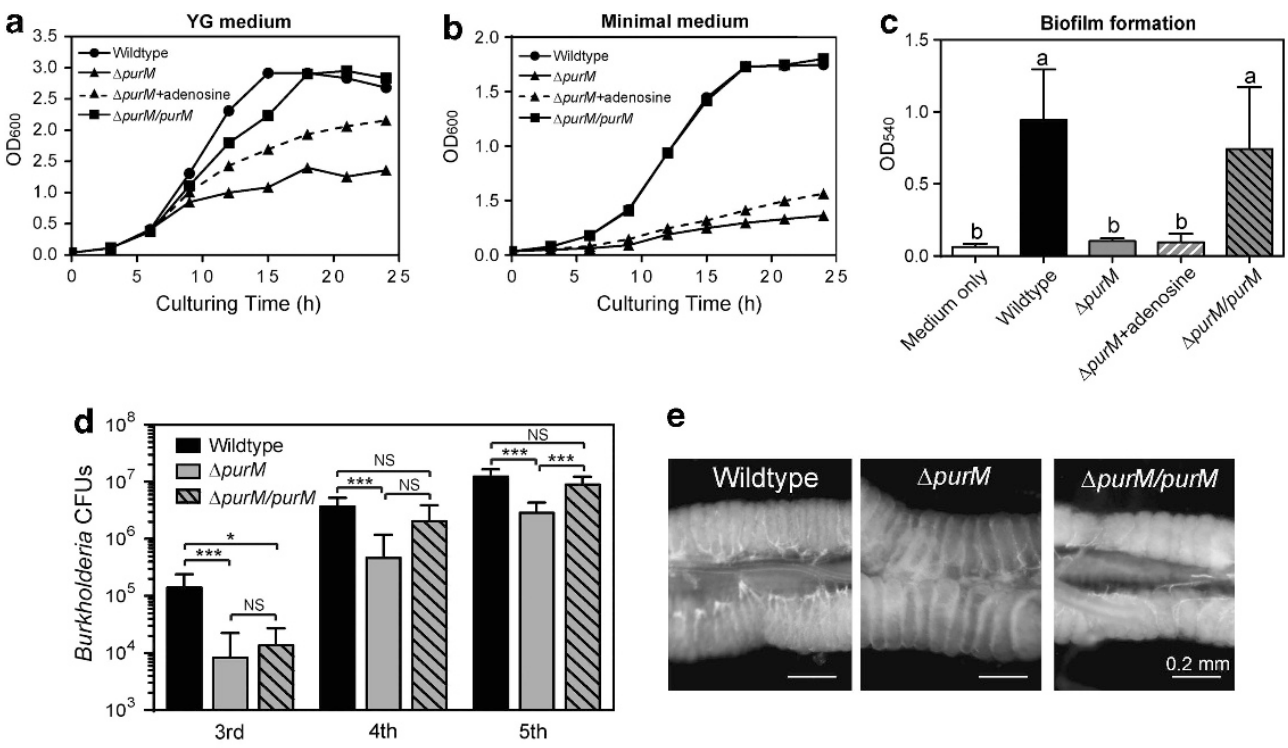

e
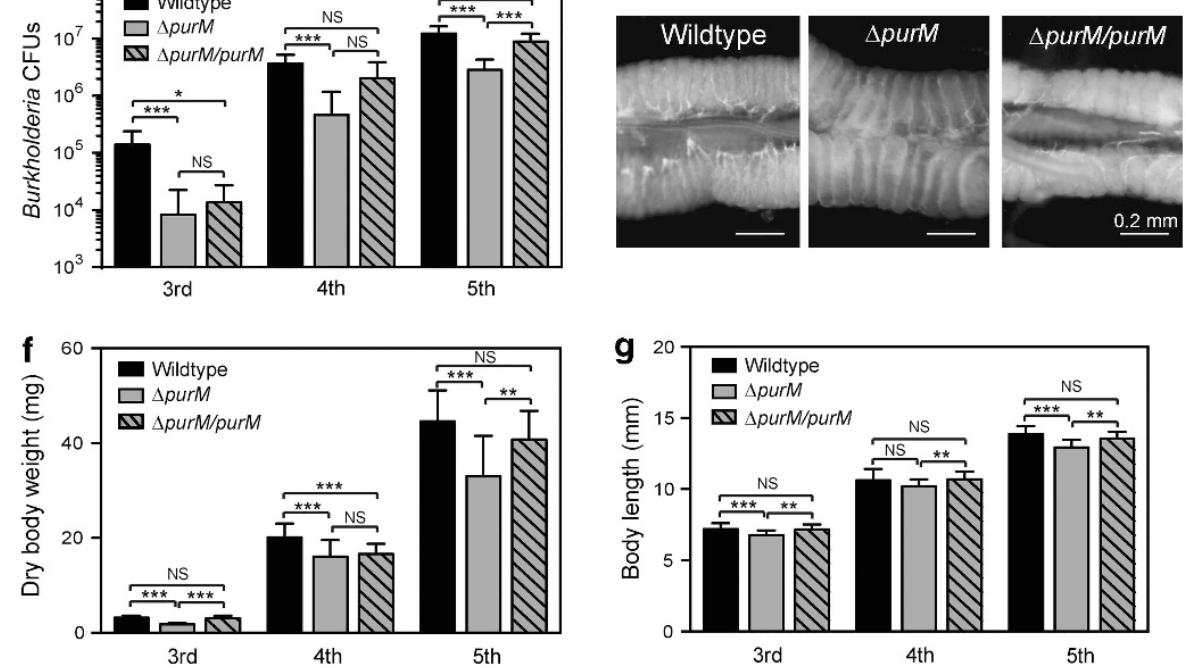

Figure 8 (a, b) Growth curves of the wildtype, $\Delta$ purM mutant and $\Delta$ purM/purM-complemented mutant strains of Burkholderia symbiont in YG medium (a) and minimal medium (b). (c) Quantitative comparison of biofilm formation by the wildtype, $\Delta$ purM mutant and $\Delta$ purM/purM-complemented mutant strains of Burkholderia symbiont cultured in YG medium. Different letters (a, b) on the top of the columns indicate statistically significant differences $(P<0.05$; one-way ANOVA with Tukey's correction). In (a-c) adenosine was added to the media to examine the auxotrophic phenotype of $\Delta$ purM. (d) Infection titers of the wildtype, $\Delta$ purM mutant, and $\Delta$ purM/ purM-complemented mutant strains of Burkholderia symbiont in midgut M4 region. Means and standard deviations ( $n=10)$ are shown. Asterisks indicate statistically significant differences (unpaired $t$-test; ${ }^{*} P<0.001$ and ${ }^{* * *} P<0.0001$ ). NS indicates no significant difference. (e) Light microscopic images of the midgut M4 region dissected from fifth-instar nymphs of $R$. pedestris infected with the wildtype, $\Delta$ purM mutant or $\Delta$ purM/purM-complemented mutant strains of Burkholderia symbiont. (f, g) Fitness values of the wildtype, $\Delta$ purM- or $\Delta$ purM/purM-infected insects as determined by body length (f) and dry body weight (g). Means and standard deviations $(n=30)$ are shown. Asterisks indicate statistically significant differences (unpaired $t$-test; ${ }^{* *} P<0.001$ and $\left.{ }^{* * *} P<0.0001\right)$. NS indicates no significant difference.

source and may therefore not support the growth of the purL mutant. Second, the defect in biofilm formation was another in vitro phenotype exhibited by the purL mutant (Figure 2c). Bacterial biofilm formation is one of the adaptation mechanisms to various environments and has been reported to be required for chronic infections and symbioses (Costerton et al., 2003; Ramey et al., 2004; Visick, 2009; An and Grewal, 2010; Burmølle et al., 2010; Morris and Visick, 2010; Rinaudi and Giordano, 2010; Maltz et al., 2012). In squid-Vibrio symbiosis, Vibrio fischeri uses a transient biofilm to colonize its host, Euprymna scolope (Visick, 2009; Morris and Visick, 2010). Although it has not yet been determined whether Burkholderia requires biofilm to enter and grow in the midgut M4 crypts, it is plausible that biofilm may support proper colonization and maintenance of the Burkholderia symbiont in the host symbiotic organ. Lastly, an unknown phenotype of the purL mutant may be a critical reason for the low infection density of the purL mutant in the host midgut. As the purL mutation interferes with purine biosynthesis and changes the purine level in the mutant cells, many pathways utilizing purine-derived molecules may be affected by the purL mutation. As shown in Figure 6i, the purL mutant exhibits deformed cell shape in the midgut M4 crypts. As the purL mutant grown in nutrientdeficient media is normal in cell shape (data not shown), it is possible that some host factors in the M4 crypt cavity may induce the cell deformation observed in the purL mutant. Such deformation supports the notion of a possible involvement of unknown phenotypes other than growth or biofilm defects. Based on these in vitro data, the accommodation defect of the purL mutant in the Riptortus host is most likely caused not by a single factor but rather a complex combination of factors. 
Properties of purL mutants in other symbiotic systems As in many pathogenic bacteria where purine auxotrophy is known to attenuate infection (Mahan et al., 1993), purL gene has also been recognized as a crucial gene in some symbiotic bacteria. For example, purL mutants of Sinorhizobium fredii failed to form nodules or elicit pseudonodules on soybean and other leguminous plants, and different levels of purL expression affected the ability of competitive nodule formation (Buendia-Claveria et al., 2003; Xie et al., 2006, 2009). Transposon-inserted Bradyrhizobium mutants-deficient in purL and other purine synthesis genes showed either nodulation deficiency or pseudonodule formation on Aeschynomene host plants (Giraud et al., 2007). In nematode-Photorhabdus symbiosis, symbiont mutants-deficient in purL were unable to form biofilm in vitro and exhibited a dramatic decrease in symbiotic persistence in nematodes (An and Grewal, 2010, 2011). Considering these accumulated lines of evidence, an understanding of the molecular mechanism of the involvement of purL in symbiosis is of great importance and requires further study.

\section{Conclusion}

Genetically modified symbiont mutants are classified into three broad classes based on their defects: initiation mutants, accommodation mutants and persistence mutants (Ruby, 2008). Here, the purL mutant is the first accommodation mutant identified in the Riptortus-Burkholderia system, which is able to colonize the host midgut but fails to reach a normal infection density. Furthermore, our findings demonstrated that the accommodation defect of the purL mutant significantly affects host crypt development and fitness.

\section{Conflict of Interest}

The authors declare no conflict of interest.

\section{Acknowledgements}

This study was supported by the Global Research Laboratory (GRL) Grant of the National Research Foundation of Korea (grant number 2011-0021535) to BLL and TF. The nucleotide sequence data reported in this paper will appear in the DDBJ/EMBL/GenBank nucleotide sequence databases with the accession numbers AB817044 (purM) and AB817045 (purL).

\section{References}

An R, Grewal PS. (2010). Molecular mechanisms of persistence of mutualistic bacteria Photorhabdus in the entomopathogenic nematode host. PLoS One 5: e13154.

An R, Grewal PS. (2011). purL gene expression affects biofilm formation and symbiotic persistence of
Photorhabdus temperata in the nematode Heterorhabditis bacteriophora. Microbiology 157: 2595-2603.

Anbutsu H, Goto S, Fukatsu T. (2008). High and low temperatures differently affect infection density and vertical transmission of male-killing Spiroplasma symbionts in Drosophila hosts. Appl Environ Microbiol 74: 6053-6059.

Baumann P, Moran NA. (1997). Non-cultivable microorganisms from symbiotic associations of insects and other hosts. Antonie Van Leeuwenhoek 72: 39-48.

Buchner P. (1965). Endosymbiosis of animals with plant microorganisms. Interscience Publishers: New York, NY, USA.

Buendia-Claveria AM, Moussaid A, Ollero FJ, Vinardell JM, Torres A, Moreno J et al. (2003). A purL mutant of Sinorhizobium fredii HH103 is symbiotically defective and altered in its lipopolysaccharide. Microbiology 149: 1807-1818.

Burmølle M, Thomsen TR, Fazli M, Dige I, Christensen L, Homøe P et al. (2010). Biofilms in chronic infectionsa matter of opportunity-monospecies biofilms in multispecies infections. FEMS Immunol Med Microbiol 59: 324-336.

Costerton W, Veeh R, Shirtliff M, Pasmore M, Post C, Ehrlich G. (2003). The application of biofilm science to the study and control of chronic bacterial infections. J Clin Invest 112: 1466-1477.

Engelstadter J, Charlat S, Pomiankowski A, Hurst GD. (2006). The evolution of cytoplasmic incompatibility types: integrating segregation, inbreeding and outbreeding. Genetics 172: 2601-2611.

Gage DJ. (2004). Infection and invasion of roots by symbiotic, nitrogen-fixing rhizobia during nodulation of temperate legumes. Microbiol Mol Biol Rev 68: 280-300.

Giraud E, Moulin L, Vallenet D, Barbe V, Cytryn E, Avarre JC et al. (2007). Legumes symbioses: absence of Nod genes in photosynthetic bradyrhizobia. Science 316: 1307-1312.

Goodrich-Blair H, Clarke DJ. (2007). Mutualism and pathogenesis in Xenorhabdus and Photorhabdus: two roads to the same destination. Mol Microbiol 64: 260-268.

Goto S, Anbutsu H, Fukatsu T. (2006). Asymmetrical interactions between Wolbachia and Spiroplasma endosymbionts coexisting in the same insect host. Appl Environ Microbiol 72: 4805-4810.

Ikeda T, Ishikawa H, Sasaki T. (2003). Infection density of Wolbachia and level of cytoplasmic incompatibility in the Mediterranean flour moth, Ephestia kuehniella. J Invertebr Pathol 84: 1-5.

Kikuchi Y, Fukatsu T. (2013). Live imaging of symbiosis: spatiotemporal infection dynamics of GFP-labeled Burkholderia symbiont in the bean bug Riptortus pedestris. Mol Ecol (in press).

Kikuchi Y, Meng XY, Fukatsu T. (2005). Gut symbiotic bacteria of the genus Burkholderia in the broadheaded bugs Riptortus clavatus and Leptocorisa chinensis (Heteroptera: Alydidae). Appl Environ Microbiol 71: 4035-4043.

Kikuchi Y, Hosokawa T, Fukatsu T. (2007). Insect-microbe mutualism without vertical transmission: a stinkbug acquires a beneficial gut symbiont from the environment every generation. Appl Environ Microbiol 73: 4308-4316.

Kikuchi Y, Hosokawa T, Fukatsu T. (2011a). An ancient but promiscuous host-symbiont association between 
Burkholderia gut symbionts and their heteropteran hosts. ISME J 5: 446-460.

Kikuchi Y, Hosokawa T, Fukatsu T. (2011b). Specific developmental window for establishment of an insect-microbe gut symbiosis. Appl Environ Microbiol 77: 4075-4081.

Kim JK, Lee HJ, Kikuchi Y, Kitagawa W, Nikoh N, Fukatsu T et al. (2013a). Bacterial cell wall synthesis gene uppP is required for Burkholderia colonization of the stinkbug gut. Appl Environ Microbiol 79: 4879-4886.

Kim JK, Won YJ, Nikoh N, Nakayama H, Han SH, Kikuchi Y et al. (2013b). Polyester synthesis genes associated with stress resistance are involved in an insect-bacterium symbiosis. Proc Natl Acad Sci USA 110: E2381-2389.

Koga R, Tsuchida T, Fukatsu T. (2003). Changing partners in an obligate symbiosis: a facultative endosymbiont can compensate for loss of the essential endosymbiont Buchnera in an aphid. Proc Biol Sci 270: 2543-2550.

Kondo N, Shimada M, Fukatsu T. (2005). Infection density of Wolbachia endosymbiont affected by co-infection and host genotype. Biol Lett 1: 488-491.

Laemmli UK. (1970). Cleavage of structural proteins during the assembly of the head of bacteriophage T4. Nature 227: 680-685.

Mahan MJ, Slauch JM, Mekalanos JJ. (1993). Selection of bacterial virulence genes that are specifically induced in host tissues. Science 259: 686-688.

Maltz MA, Weiss BL, O’Neill M, Wu Y, Aksoy S. (2012). OmpA-mediated biofilm formation is essential for the commensal bacterium Sodalis glossinidius to colonize the tsetse fly gut. Appl Environ Microbiol 78: 7760-7768.

McGraw EA, Merritt DJ, Droller JN, O’Neill SL. (2002). Wolbachia density and virulence attenuation after transfer into a novel host. Proc Natl Acad Sci USA 99: 2918-2923.

Moran NA, McCutcheon JP, Nakabachi A. (2008). Genomics and evolution of heritable bacterial symbionts. Annu Rev Genet 42: 165-190.

Morris AR, Visick KL. (2010). Control of biofilm formation and colonization in Vibrio fischeri: a role for partner switching? Environ Microbiol 12: 2051-2059.

Mouton L, Henri H, Bouletreau M, Vavre F. (2003). Strainspecific regulation of intracellular Wolbachia density in multiply infected insects. Mol Ecol 12: 3459-3465.

Mouton L, Dedeine F, Henri H, Bouletreau M, Profizi N, Vavre F. (2004). Virulence, multiple infections and regulation of symbiotic population in the WolbachiaAsobara tabida symbiosis. Genetics 168: 181-189.

Mouton L, Henri H, Charif D, Bouletreau M, Vavre F. (2007). Interaction between host genotype and environmental conditions affects bacterial density in Wolbachia symbiosis. Biol Lett 3: 210-213.

Nyholm SV, McFall-Ngai MJ. (2004). The winnowing: establishing the squid-vibrio symbiosis. Nat Rev Microbiol 2: 632-642.

Oliver KM, Degnan PH, Burke GR, Moran NA. (2010). Facultative symbionts in aphids and the horizontal transfer of ecologically important traits. Annu Rev Entomol 55: 247-266.

Pontes MH, Dale C. (2006). Culture and manipulation of insect facultative symbionts. Trends Microbiol 14: $406-412$.

Ramey BE, Koutsoudis M, von Bodman SB, Fuqua C. (2004). Biofilm formation in plant-microbe associations. Curr Opin Microbiol 7: 602-609.

Rinaudi LV, Giordano W. (2010). An integrated view of biofilm formation in rhizobia. FEMS Microbiol Lett 304: 1-11.

Ruby EG. (2008). Symbiotic conversations are revealed under genetic interrogation. Nat Rev Microbiol 6: $752-762$.

Sakurai M, Koga R, Tsuchida T, Meng XY, Fukatsu T. (2005). Rickettsia symbiont in the pea aphid Acyrthosiphon pisum: novel cellular tropism, effect on host fitness, and interaction with the essential symbiont. Buchnera. Appl Environ Microbiol 71: 4069-4075.

Schägger H. (2006). Tricine-SDS-PAGE. Nat Protoc 1: 16-22.

Vautrin E, Vavre F. (2009). Interactions between vertically transmitted symbionts: cooperation or conflict? Trends Microbiol 17: 95-99.

Visick KL. (2009). An intricate network of regulators controls biofilm formation and colonization by Vibrio fischeri. Mol Microbiol 74: 782-789.

Werren JH, Baldo L, Clark ME. (2008). Wolbachia: master manipulators of invertebrate biology. Nat Rev Microbiol 6: 741-751.

Xie B, Chen DS, Zhou K, Xie YQ, Li YG, Hu GY et al. (2006). Symbiotic abilities of Sinorhizobium fredii with modified expression of purL. Appl Microbiol Biotechnol 71: 505-514.

Xie B, Chen D, Cheng G, Ying Z, Xie F, Li Y et al. (2009). Effects of the purL gene expression level on the competitive nodulation ability of Sinorhizobium fredii. Curr Microbiol 59: 193-198.

Supplementary Information accompanies this paper on The ISME Journal website (http://www.nature.com/ismej) 